\title{
Analyse critique ou lecture critique des articles médicaux : quelle cible choisir pour l'enseignement et l'évaluation ?
}

France, les modalités d'évaluation des étudiants en M édecine à la fin du $2^{\mathrm{e}}$ cycle des études médicales, doivent être modifiées en 2004. Le Concours del'I nternat sera supprimé. Un examen classant national sera passé par tous les étudiants pour accéder au $3^{\mathrm{e}}$ cycle des études médicales. Cet examen, dont les modalités ne sont pas définitivement arrêtées, pourrait comporter trois types d'épreuves:

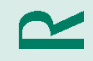

- D es dossiers éval uant les capacités cliniques et privilégiant la transversalité.

- Des questions rédactionnel les courtes explorant la capacité à maîtriser les méthodes diagnostiques et thérapeutiques courantes, ainsi que les contraintes médico-légales et médico-sociales.

- U ne analyse critique d'articles appréciant la capacité à l'auto-formation.

Ce dernier type d'épreuve comporterait l'analyse d'un article médical sur des essais thérapeutiques ou des études épidémiologiques; son poids sur la note totale serait de $20 \%$.

Cette épreuve d'analyse critique d'articles suscite des craintes de la part des enseignants et probable ment des étudiants. II ne sagit pas d'un simple mouvement d'humeur face à une innovation, mais d'une réelle inquiétude dont les fondements sont de natures diverses Quelles sont donc les raisons de cette inquiétude?

En premier lieu, il existe une ambiguïté sur la définition de la terminologie " analyse critique d'articles». En effet, pour la majorité des enseignants habitués à être experts pour la révision d'articles avant publication dans des revues scientifiques, I'analyse critique d'articles est un exercice difficile. Les éditeurs des journaux et des revues médicales sélectionnent les spécialistes les plus compétents pour leur soumettre à critique les articles qu'ils reçoivent en vue de publication. L'analyse critique est une tâche qui exige une grande connaissance du sujet, de solides bases en méthodologie, une expérience clinique établie de longue date, une grande rigueur de raisonnement et un souci constant de l'apport d'un niveau de preuve suffisant. Cet exercice demande beaucoup de temps, nécessite la lecture et la relecture d'articles précédemment publiés sur le même sujet, l'interrogation éventuelle d'autres coll ègues mi eux instruits sur tel ou tel point de l'étude.

Cet exercice, en raison de ses exigences, ne paraît pas adapté au niveau d'étudiants du $2^{\mathrm{e}}$ cycle des études médicales. En revanche, sans vouloir jouer sur les mots, la formation à la « lecture critique d'articles » peut être intégrée précocement dans le cursus des études médicales. Elle constitue un des moyens propices pour une autoformation pendant la formation initiale, mais auss pour toute la durée de la vie professionnelle. II et par consé quent urgent que soit levée l'ambiguité entre la « critique d'articles», véritable travail réservéaux experts, et la « lecture critique », compétence qui requiert des habiletés spécifiques qui sont rapportées dans un article publié dans ce numéro de Pédagogie M édicale ${ }^{1}$. Dans leur article, $N$. Audet et $H$. Ledèret font part de leurs recherches à propos des habilités requises pour la lecture critique en M édecine. Bien que leurs propos concernent les médecins praticiens en exercice, bon nombre de propositions pourront être retenues pour les étudiants.

En deuxième lieu, les enseignants pensent qu'ils sont habilités à évaluer ce qu'ils savent enseigner. Or ceux-ci n'ont pas, pour la plupart, une connaissance des méthodes pour guider l'étudiant dans l'acquisition des habiletés pour une lecture critique d'articles. N ous pouvons le regretter, mais il sagit d'un fait indiscutable. Ceux qui auraient la curiosité de rechercher dans la littérature médicale des références pour guider leur approche de ce problème, risquent d'être déçus. Deux articles récents, publiés respectivement dans 
Medical Education ${ }^{2}$ et Academic M edicine, rapportent une analyse systématique des expé riences publiées sur ce sujet. Ces éudes révèlent que les expériences publiées sont peu nombreuses; elles concernent la formation de résidents (internes) en M édecine générale ou en spécialité, ou de praticiens en formation continue Les modalités de formation sont très variables, maisil s'agit majoritairement d'exercices pratiques en petits groupes, allant de 5 à 17 sessions d'une durée de 30 à 90 minutes. Enfin, l'une des études suggère que l'impact de cette formation à la lecture critique se solde par des résultats modestes avec une amélioration des scores d'évaluation des participants de 0 à $23 \%{ }^{3}$.

$\simeq$ II faut donc que, sans délai, certains enseignants prennent en charge, dans chaque faculté, les mis sions de définir les objectifs spécifiques de la formation à la lecture critique d'articles, de conceptuali ser des méthodes adéquates pour cette formation et d'organiser matériellement l'enseignement. Les concepts de base peuvent être enseignés dès le début du $2^{2}$ cycle, qu'il sagisse des méthodes d'enquêtes épidémiologiques, d'évaluation des procédures diagnostiques et de dépistage, des critères de qualité souhaitables des instruments de mesure, des plans de recherche clinique ou thérapeutique, des essais cliniques et thérapeutiques, des tests courants en biostatistique. L'enseignant devrait proposer aux étudiants des exercices pratiques en petits groupes, à l'aide de textes conformes aux critères de qualité d'un bon article et de textes comportant des erreurs ou des biais, afin d'aider les étudiants à percevoir les qualités et les défauts des articles médicaux, comme cela a été suggéré dans un article récent ${ }^{4}$. Enfin, au cours de ces exercices pratiques, les étudiants devraient être entraînés à la rédaction de résumés, à la lecture de tableaux et figures et à l'examen des références bibliographiques.

En trois ième lieu, les enseignants et les étudiants désirent ardemment connaître les « règles du jeu » de l'évaluation. Chacun peut admettre que l'évaluation doit être en accord avec les objectifs de I'enseignement (ce qui souligne l'importance de les énoncer). Elle doit être fidèle et sans ambiguïté, d'autant qu'il s'agit d'un examen classant qui aura une influence sur les possibilités de choix d'orientation future des étudiants, et probable ment sur l'évaluation des Facultés. Elle doit enfin être praticable en tenant compte du fait qu'elle sadresse à des milliers de candidats.

En conclusion, on peut penser qu'au sein des multiples possibilités offertes grâce aux progrès de la communication, la lecture demeurera une action de formation continue privilégiée par les médecins pour entretenir et développer leurs compétences. II paraît indispensable de préparer très tôt les étudiantsà la lecture critique d'articles. Ayant acquis compétence et expérience dans ce domaine, ils seront alors capables de faire une bonne analyse critique des publications médicales. II est toutefois permis de sinterroger sur la position volontariste des responsablesnationaux qui, en incluant l'anaIyse critique d'articles dans l'examen classant validant, alors que nous ne disposons pas encore de méthodes valides et fidèles pour évaluer cette capacité, ont pris des risques importants qui pourraient altérer l'équité de l'examen national et aboutir à des situations de contentieux.

$$
\begin{aligned}
& \text { Jean-Paul Fillastre* - Raymond Colin }{ }^{*} \\
& * \text { jeanpaul.fillastre@chu-rouen.fr } \\
& * * \text { raymond.colin@chu-rouen.fr }
\end{aligned}
$$

\section{Bibliographie}

1. Audet N N, Leclère H. Les habiletés requises pour la lecture critique en médecine : un cadre de référence issu d'une recension desécrits. Pédagogie M édicale 2001 ; 4 : 206-212.

2. Taylor $R$, Reves $B$, Ewings $P$, Binns S, Keast J, M ears R. A systematic review of the effectiveness of critical appraisal skills training for clinicians. M edical Education $2000 ; 34: 120-25$.

3. Green M L. G raduate medical education training in clinical epidemiology, critical appraisal and evidence-based medicine : a critical review of curricula. Academic M edicine $1999 ; 74: 686-94$.

4. N eville AJ, Reiter HI, Eva DW, N orman GR. Critical appraisal turkey shoot : Linking critical appraisal to cli nical decision making. Academic M edicine $2000 ; 75$ oct suppl : 587-89. 